\title{
Static and Dynamic User Portraits
}

\author{
Ko-Hsun Huang, ${ }^{1,2}$ Yi-Shin Deng, ${ }^{3}$ and Ming-Chuen Chuang ${ }^{1}$ \\ ${ }^{1}$ Institute of Applied Arts, National Chiao-Tung University, 1001 University Road, Hsinchu 300, Taiwan \\ ${ }^{2}$ Madeira Interactive Technologies Institute, Caminho da Penteada, 9020-105 Funchal, Portugal \\ ${ }^{3}$ Institute of Creative Industrial Design, National Cheng Kung University, 1 University Road, Tainan City 701, Taiwan
}

Correspondence should be addressed to Ko-Hsun Huang, kohsun.huang@gmail.com

Received 18 May 2012; Revised 12 September 2012; Accepted 13 October 2012

Academic Editor: Bill Kapralos

Copyright ( $\odot 2012$ Ko-Hsun Huang et al. This is an open access article distributed under the Creative Commons Attribution License, which permits unrestricted use, distribution, and reproduction in any medium, provided the original work is properly cited.

User modeling and profiling has been used to evaluate systems and predict user behaviors for a considerable time. Models and profiles are generally constructed based on studies of users' behavior patterns, cognitive characteristics, or demographic data and provide an efficient way to present users' preferences and interests. However, such modeling focuses on users' interactions with a system and cannot support complicated social interaction, which is the emerging focus of serious games, educational hypermedia systems, experience, and service design. On the other hand, personas are used to portray and represent different groups and types of users and help designers propose suitable solutions in iterative design processes. However, clear guidelines and research approaches for developing useful personas for large-scale and complex social networks have not been well established. In this research, we reflect on three different design studies related to social interaction, experience, and cross-platform service design to discuss multiple ways of identifying both direct users and invisible users in design research. In addition, research methods and attributes to portray users are discussed.

\section{Introduction}

Understanding target users is considered a basic step towards developing good products and services. In traditional industrial design, marketing, city planning, and environmental design, a wide range of research methods, including surveys, field studies, interviews, and focus groups, have been used with the clear purpose of identifying target audiences' preferences and needs [1-4]. In system and software development, usability evaluation and user-centered design methods, such participatory design, contextual inquiry, or ethnographic techniques, have also been well accepted and applied to better understand end users' knowledge background, behaviors, cognitive processes, and requirements [5-7].

To support rapid IT development and iterative design processes, it has become important to have clear images and models to represent end users: ideally models which can be reused and reapplied in the development of different products and services [8]. In software engineering, user modeling has focused on having an internal representation of users, which includes information such as background knowledge, preferences, and the ways that users interact with systems [9-11]. This type of user modeling or profiling can be used to design serious games, educational training, and learning systems or to evaluate systems by simulating different types of users [11-13]. Beyond traditional user cognitive models, much work has extended the modeling variables to cover users' previous computing experiences, personality traits, and background context for educational applications [11, 14]. Hothi and Hall [15] have addressed $\mathrm{SaD}$ (static and dynamic) user modeling, which focuses on both static information such as gender and age, and dynamic information such as diverse computing experiences and personality. They considered that such dynamic user data could be used to make a system dynamically adapt to an individual user. Karampiperis and Sampson [14] have highlighted two distinct submodels in adaptive educational hypermedia development: a frequently updated model to represent learners' knowledge space and a static model to represent learners' cognitive characteristics and learning preferences $[11,14,16]$. 
With a purpose similar to that of user modeling, personas have also been used to portray user types in serious games, adaptive training systems, communication, and experience design $[17,18]$. The original concept of personas is to create fictional characters to represent different user and consumer types by describing their tastes, perceptions, possible reactions, and attitudes towards a certain product, service, or brand [18-20]. There are several significant benefits to having personas in the design process, such as providing all members of the development team a clear and common image of the target audience, evaluating if design solutions meet users' needs, and enhancing practitioners empathy on a certain type of user $[18,21]$. For instance, Antle [22] has argued that most successful products for children are neither goal- nor task-oriented but meet specific needs of children in a particular age range. She has highlighted that child-personas of more experiential contexts can provide a way to incorporate concepts from developmental psychology into design and allow archetypical users to be presented throughout the design process for technology-enhanced educational systems [22-24]. To design roles and scenarios for serious games and game-based adaptive training systems, Raybourn [17] has also suggested using personas to guide the design process and to help game designers and game writers develop realistic or believable roles for players.

Although both user models and personas can offer various benefits in different phases of iterative design, including initial concept development, user testing, and redesign cycles, there are several problems associated with their application in complex designs of social interactions, experiences, and services. First, user-modeling methodology has been developed with a focus on human-computer interaction and centers on task-oriented analysis of behavior patterns, cognitive processes, and demographic data. The approaches and modeling can reveal detailed information about the ways that a user interacts with a system, but are incapable of taking large-scale social contexts into account [25-27]. Such modeling cannot account for subtle social interactions and communication between people, which is the emerging focus of serious games, adaptive hypermedia, and learning networks $[28,29]$. Secondly, the information that a traditional persona offers is also limited to a few attributes. Although a person's sociocultural background and economic status may be considered, detailed information such as one's relationships with communities, products, and technologies, are generally absent. In addition, several researchers have criticized persona development for lacking scientific process and clear guidelines, which decrease the method's reliability and representability [30-33]. Both user modeling and personas restrict their scope by overlooking activity contexts; user modeling only focuses on direct users of systems, and personas mainly represent target and potential consumers, rather than capturing and revealing all participants involved in the activities of interest.

Much work has shown that having social perspectives and understanding nuanced social interaction will be the most challenging but necessary topic in today's experience and social media design $[4,7,17]$. In addition, much work has shown that enhancing social identity and applying social influence can help practitioners and designers develop systems for learning, behavior changing, and other social purposes $[28,34,35]$. Furthermore, design strategies such as applying CASA (Computer as Social Actor) in technologyenhanced education also require a thorough understanding of learners' sociocultural backgrounds $[36,37]$.

This paper discusses current methods, approaches, and frameworks applied in design research, including several user-centered approaches, design frameworks, and models developed for studying cultures. We highlight the strengths and shortcomings of these methods when applied to capture different levels of user information, from detailed interactions to sociocultural backgrounds [3-5]. Examples from user studies in three design projects are used to illustrate that the different levels of information help identify user types and build user portraits. In each case, multiple ethnographical techniques were applied, such as shadowing, in-depth interviews, grounded theory [38, 39], work modeling [40], and social activity modeling [4], to reveal the complicated involvement of different types of participants in social activities and product usage.

We reflect on the three case studies to recommend suitable user study methods for supporting large-scale services, adaptive systems, and development of serious games, as well as necessary information to gather when portraying users. By understanding participants' relationships and roles within a broader context, we also derive insights for identifying invisible users and potential users and the importance of understanding different users' motives and concerns.

\section{Methods and Frameworks}

Emerging areas of interest in IT development, such as serious games, adaptive systems, and experience design, are concerned with large complex communities and diverse generations. Large-scale services such as e-government and social media inevitably have to support people's social activities in the coming future. According to the central concept of ethnomethodology, people are intelligent and creative, and with ad hoc practices, they can apply their knowledge across domains and act in different contexts [41, 42]. It is the methods used by people to apply knowledge and their values and attitudes, which matter and can help scholars to predict further actions, reactions, and acceptance towards a certain system or service. Instead of looking into what people actually perform, understanding background motives is essential for developing more adaptive and flexible systems and services for greater social purposes in the future.

2.1. Models for Social and Cultural Studies. In social science, the strategies to study a culture include traditional anthropological approaches, such as participant observations with long-term involvement of the fields and interpretative approaches, in which researchers collect, conceptualize, and induce the concepts through diverse methods. For years, numerous discussions and debates have been held about ways to generate more solid and scientific results while 
applying these methods and approaches. Many researchers have focused on measurements and standards for improving the validity and reliability of qualitative research [43-45]. For instance, Glaser and Strauss [38] developed a grounded theory in which researchers analyze data and generate a theory repeatedly until they can thoroughly explain and describe the phenomenon. In this iterative process, Strauss placed emphasis on improving reliability and validation criteria in a systematic way $[38,39,46]$. However, in IT and product design practices, while designers and developers apply qualitative approaches, they tend to analyze the situations by using models as guidelines and check lists, and the research results generally only support short-term and inner group usages and have no impact on further related studies.

Applying the above-mentioned positivist or interpretivist approaches to gain knowledge about society and culture is very time consuming and requires experienced researchers to collect and analyze raw data. Therefore, to reduce the cost and to gain insights more efficiently, there are numerous theoretical frameworks and models developed in different domains. For instance, since the 1980s, researchers in management and leadership have discussed numerous models for understanding organizational cultures [47-51]. Most of these works have the same purpose, either to optimize the organizational process or to reduce internal conflicts within organizations.

In the fields of management and leadership, many scholars have taken similar approaches to identify cultures-firstly recognizing a culture's representative characteristics and then categorizing them into types. These researchers generally take an organizational point of view and focus on structures, power distribution, and divisions of labor $[48,52,53]$. The earliest work is Harrison's organization ideologies, in which there are four types of cultures highlighted by their typical features $[52,54]$. This classification refers to power-, role-, task-, and person-oriented cultures, respectively, standing for centralized power, hierarchical structure, team support, and individual achievement [52, 55, 56]. Harrison's work has had a great impact on later studies of organizational culture, in which the power distribution and organizational formalization have become the basic criteria to locate a culture [52]. Furthermore, the ways that an organization or a community responds to outside influence and different situations, the flexibility and stability of organizational structures, and the forms of attention have also been widely discussed [48, 53, 57].

In contrast to categorizing cultures into types, many scholars focus on finding cultural concepts and patterns for intercultural studies. The earliest works are Hall's books, in which he identified two dimensions of culture, including the high- and low-context communication and polychromic versus monochromic time orientation [58, 59]. The high-low context concept is used to characterize information transaction and interpersonal communication in cultures, and the second concept is concerned with the way in which people structure their time, tasks, and schedules. Moreover, to deal with cross-national issues in the functioning of organizations, Hofstede [60] indicated the differences between studying national cultures and organizational cultures where national cultures differ primarily in their values, but organizational cultures turn out to differ mainly in their practices. He identified six different dimensions of organizational cultures and claimed five other national cultural dimensions, including power distance, uncertainty avoidance, individualism versus collectivism, masculinity versus femininity, and long- versus short-term orientation $[60,61]$. For understanding cultural diversity in global business, Trompenaars and Hampden-Turner [62] also developed a model of culture with seven dimensions, including universalism versus particularism, individualism versus collectivism, neutral versus emotional, and specific versus diffuse.

Instead of classifying existent international or organizational cultures into types, some researchers have focused on developing models for analyzing and understanding the culture of a particular group or organization. For instance, Schein [63] identified three fundamental levels at which culture manifests itself: observable artifacts, values, and basic underlying assumptions. Here, artifacts refer to physical layouts, manners, atmosphere, and phenomena that people can directly feel and observe, and values refer to members' norms, ideologies, and philosophies. The basic assumptions are those taken-for-granted, underlying and usually unconscious aspects that people have and share within groups, and they normally can determine people's perceptions, thought processes, and behaviors [63, 64]. Closely resembling Schein's three levels, Hofstede placed four manifestations of cultures at different levels of depth, namely symbols, heroes, rituals, and values [57, 60]. Rousseau [49] also detailed the nature of the cultural construct and its theoretical roots and layered these cultural elements according to subjectivity and accessibility. His five layers of culture, from outside in, are artifacts, patterns of behavior, behavioral norms, values, and unconscious fundamental assumptions. This type of layered cultural model also appears in many other researchers' works, in which basic assumptions and values are generally placed at the core layer(s), and then are encircled by beliefs, attitudes, rituals, behaviors, and then artifacts $[65,66]$.

There is one extreme example of the ambitious objective to combine all aforementioned concepts into one single model. Considering cultural impact on the implementation of enterprise resource planning (ERP), Krumbholz and Maiden [67] developed a metaschema for modeling culture. They integrated the surface and the deeper manifestations of culture into common business concepts such as processes, events, and information flows, and then developed a systematic framework to analyze culture for further explanation, prediction, and replanning of different corporate and national cultures. Their framework is based on Schein's three levels of culture and a wide range of social aspects (e.g., group norms, formal philosophy, and linguistic paradigms). Influenced by globalization trends and comparative studies, they also included Hofstede's and Trompenaars and Hampden-Turner's cultural dimensions in their work $[16,50,62]$. This enormous framework has more than twenty components in three main categories. 
The first category covers common elements in business processes, such as agent, role, responsibility, and goal. The second category describes the core levels of culture, including hidden assumptions, beliefs, and values. The last category focuses on characteristics such as customs, symbols, and environments. Krumbholz and Maiden tried to cover all the important elements discussed in cultural studies. However, there are many unclear definitions and assumptions in this metaschema. For instance, social interaction is a significant component of the model, which influences and reflects one's beliefs and represents the types and ways people interact with each other. However, their work does not detail how to explain relationships between social actions, values, and beliefs nor does it suggest methodology to gain such an understanding.

The models of cultural types provide several ways to identify national or organizational cultures and have indicated the significant relationships between power structure and personal behaviors $[48,52-54]$. However, there are two major reasons why it is difficult to apply these models directly as research frameworks in other domains. Firstly, the limited cultural types oversimplify the sociocultural issues and avoid the level of depth in cultural significance. In management and leadership cases, the classification approach provides a simple and quick way to identify the cultural characteristics, but due to a lack of clear essential definitions, these models are incapable of revealing the subtle relationships and influences between the cultural components. Second, most human behaviors, especially social activities, do not have a clear objective and can be analyzed into steps and processes. With a strong management and leadership purpose, these models purely focus on work processes and the ways that people achieve their goals and seldom take individuals' motives, attitudes, and perspectives into account. The similar tendency of work and goal orientation appealed in Hofstede's global-scale survey [60, 61], which he conducted to evaluate the work values of a specific company. Although Trompenaars and Hampden-Turner [62] discussed personal attitudes towards both leisure and work situations, their results related to underlying assumptions and values were very limited. Krumbholz and Maiden [67] have tried to combine both social science theories and psychological concepts into their metaschema, but their interview results did not reflect the richness and interaction among these cultural levels and components. In addition, the numerous components involved in different perspectives are all compressed into a single model, which makes this metaschema difficult to use as a research framework or as a format for representing the final output of the research.

In social interaction and experience design, issues which are related to individual attitudes and values can often be traced back to and better understood in the light of social norms or culture. However, studies of how society and culture act as constraints for design have very different goals than traditional broad studies of culture itself. They also differ from the above-mentioned models and measurements of culture which focus purely on work and whose purposes are either to enhance organizational performance or to improve management and leadership. Due to their strong task-orientation, the methodologies proposed for these studies focus on practical and behavioral views, environments, and symbols. They overlook several cultural aspects which are important in socially motivated interactions between people, technology, and services, such as people's motives for actions and their emotional needs.

\subsection{Frameworks for Understanding Users and Usage Contexts.} In the past decades, design research of information and system development has focused on interactions between humans and machines. Based on the introduction of cognitive psychology, most studies have concentrated on mental processes and information flows, with the scope being achieving a task or solving a problem. Taking an example of Donald Norman's seven stages of action, which have had a lasting impact on usability engineering and industrial design, the analysis of human action is concerned with a loop of forming the goal, forming the intention, specifying an action, executing the action, perceiving the state of the world, interpreting the state of the world, and evaluating the outcome [68]. Comparing these types of mental models with microsociological theories (e.g., symbolic interactionism and dramaturgy), they all focus on the interaction between individuals and environments and discuss how people perceive and interpret the outside signals, symbols and then take an action [69].

The scope of design research in technology development expanded during the 1990s from task analysis to more complex activity analysis. For understanding larger-scale usage contexts and supporting user experience design, many ethnographical research methods were introduced in design practices, such as interviews and long-term involvement observations [70-72]. In addition, to speed up design cycles, many analytic frameworks and models were introduced and developed to make user research more efficient, cheaper, and deeper. These frameworks, such as AEIOU (activity, environment, interaction, object, and user), POEMS (people, objects, environments, message, and services), Ax4 (atmosphere, actors, activities, and artifacts), activity theory, and contextual design methodology, have been discussed and applied in various domains with great success $[40,70,73-$ 75]. Most of the frameworks provide clear guidelines and dimensions for investigating the entire activity context, including practical behaviors and actions, related objects and environment settings, and information content, as well as taking into account relationships among people.

Among these research frameworks, contextual design methodology [40] and activity theory have their specific advantages for both system development and other design practices. Contextual design methodology was developed based on research techniques of ethnography and was influenced by the development of participatory design techniques in the 1980s and 1990s. It has a special purpose to help researchers and designers identify domain problems in rapid design cycles, especially for software and hardware redesign and usability evaluation. To help researchers and designers convey their domain knowledge, thoughts, and ideas, Beyer and Holtzblatt [40] developed five work models as a tangible 
representation for issues in different dimensions, including the flow model, cultural model, sequence model, artifact model, and physical model. Furthermore, for developing a successful system, which can "fit with the customer's culture, make conforming to policy easy and reduce friction and irritation in the workplace," Beyer and Holtzblatt [40] addressed the importance of understanding organizational culture. Their cultural model highlights cultural influences among individuals, groups, and the organization and also helps researchers identify invisible power, preferences, values, and emotions. Although the cultural model concentrates on representing organizational culture, it still shows individuals' opinions and attitudes to some extent.

Activity theory, with its roots in the 1930s Soviet culturalhistorical psychology, was introduced and adapted into HCI and CSCW as a lens in ethnographic research $[73,76,77]$. To understand the mental capability of a single individual, activity theory considers a "goal-directed" activity as the unit of analysis and provides an analytical framework to describe activities with three hierarchical constructs: subject, object, and tool. In the theory, activities are described by how a single individual (subject) achieves a goal (object) through tools, and using tools reveals the details of both physical interactions and mental processes. Under this framework, a complex activity can be broken down into action or operation levels and can be analyzed from both behavioral and psychological viewpoints. To deal with multiuser systems and collaborative work, Engeström [73, 78] later proposed an extended schema for activity theory with additional constructs of community (people who share the same goal), rules, and division of labor. Engeström's schema makes activity theory very useful in groupware and social media design $[79,80]$.

With a strong intention to bridge the gap between subjective-to-objective and macro-to-micro concerns in design study, MultiLevel Social Activity Model (MLSAM) was proposed by Huang and Deng $[4,81]$. There are two basic arguments behind the concepts. First, they argued that social behaviors are deeply localized and historical on the account of cultural background. They showed that the traditional customs perform a social function by creating cohesiveness in families and by offering a habitual practice that can be passed on from one generation to another. The social activity not only reflects norms and common values of the society, but also presents various inherent characteristics of the cultural context. Secondly, they argued that people have great agency and creativity to fulfill needs and achieve purposes in a variety of ways. Traditional field observations and user studies, which focus on a limited scope and settings, can only reveal partial results of how people perform to achieve their goals. Hence, their model emphasizes the necessity to identify people's motives and attitudes, which comparing to actual actions, are more permanent and static, and could be considered in further design for different platforms and services.

From an integrated sociological viewpoint, individuals' behaviors are not only encouraged, but also constrained by norms, religions, and sociocultural backgrounds. To well support hypermedia and adaptive systems for social purposes in the future, design research needs to extend from traditional usability evaluation and task-oriented studies to a larger-scale sociocultural scope. Therefore, an in-depth research approach is needed to answer to the complexity of social interactions. The following section presents three case studies of applying both contextual design [40] and multilevel social activity model $[4,81]$ in design practices and shows the benefits of applying multiple research methods to portray users.

\section{Design Studies}

To better support product and service design in the future, we applied multiple user study methods to uncover the complexity of participants' perspectives, interactions, and attitudes within different types of activities: a traditional ritual of a Taiwanese tea ceremony, Taiwanese teenagers' social activities, and technology use, and sports watching in Southern Europe. The first study is selected to show how well-accepted software design methods can be used together with the multilevel social activity model $[4,81]$, to capture more user information within a social event, a cultural tradition. The second case study is selected to discuss if this integrated approach can also be applied to investigate a complex physical and virtual social context. The third case study is a service design, in which the same approach is applied to identify different user types and help designers and practitioners make design decisions on developing adaptive and cross-platform systems. Contrary to what is common in traditional IT development, we identify different user types by using both the contextual design flow model [40] and the multilevel social activity model [4], to highlight participants' roles, relationships, motives, and sociocultural background.

3.1. A Traditional Social Ritual. The first case is a study of traditional tea ceremonies in Taiwan. These ceremonies have deep cultural roots and also contain complex forms of social interaction, which are typically ignored in most IT development and for which established research methodology is lacking [4]. We reflect on this case study because of its rich sociocultural backgrounds, which represent a good example to show complex social relationships and different user motives within an activity.

In this case study, we applied multiple user experience research methods, which include observations [3], contextual design work modeling [40], social activity modeling [4, 81 ], and in-depth interviews with grounded theory analysis $[38,39]$, to reveal different aspects of personal perceptions toward the overall sociocultural context of Taiwan's tea ceremony. In order to identify the value of the tea ceremony to participants from different generations, three subjects of different ages were recruited, together with their regular tea ceremony groups. The three subjects were all experts of tea ceremonies. The first subject was a forty-year-old parent with a twenty-year experience of tea ceremonies. The second subject was a retired senior citizen, with a seventeen-year experience and with an interest in learning techniques and 
knowledge of brewing tea from magazines and other connoisseurs. The third subject was a female graduate student, with a fourteen-year experience of tea ceremonies and with familiarity of the Internet and associated technologies. Each had taken part in tea ceremonies at least once per week in recent months. To observe the detailed social interaction within the activity, their family members and friends, who are regular members of the ceremonies, were also invited to participate in the sessions of observations and contextual inquiries. In total, we had ten participants.

The flow model in Figure 1(a) represents the different roles in the ceremony and their interactions. On the basis of the in-depth interviews and contextual inquiries, this flow model has been extended to portray all groups who directly or indirectly engage in a ceremony. Direct participants are the host (tea server), family members, and other friends, while indirect participants include hobbyists, tea sellers, tea producers (farmers in direct marketing), and tea connoisseurs. Although indirect participants do not actually attend ceremonies, they significantly impact the act through their close ties to the host. According to the study, the host of a ceremony is most likely a person of the middle-aged generation or the head of household. This person tends to actively exchange information and sentiments with other hobbyists and tea sellers, as well as seek out information from books, magazines, and newspapers.

While the flow model captures roles and interactions, the multilevel social activity model further reveals how participants' underlying motives and attitudes toward tea ceremony differ between generations. It also shows that attitudes and resulting behaviors are all strongly rooted in a cultural context. Both the younger generation and the elderly/middle-age generations (both groups in Figure 1(b)) are direct participants in tea ceremonies (marked in orange in Figure 1(a)). However, a comparison of the two models reveal that although the younger generation is interested in tea ceremonies and has inherited tea-drinking habits from their families, they are generally not consumers of the tea industry. They have neither contacted any other participants (i.e., tea sellers and tea farmers in direct marketing), nor do they receive information through magazines or newspapers. Instead, their primary sources of information about tea ceremonies are parents or grandparents.

The multilevel social activity model identifies different user groups by their attitudes towards activities and motives for participation. In addition to people's direct and immediate requirements, we consider that there are many hidden reasons for people to engage in a social activity that may be overlooked by methodologies that do not go deep enough. For instance, the social activity model reveals that the elderly have positive attitudes toward tea ceremonies potentially reflecting the awareness of Westernization, while the younger generation is more attracted by the health benefits associated with drinking tea.

An understanding of differences in user groups' cultural backgrounds and underlying motives can help designers make better decisions, in particular in design for social activities and communication. For instance, knowledge of cultural features, for example, pouring a cup of tea to convey esteem and respect during a tea ceremony, can further lead to more accurate portraits of users and better predictions of user behaviors. However, it is difficult to gain this knowledge using only activity- and usage-centered design methods. Instead, the case studies indicate that the knowledge can be gained from a historical and broader-context approach.

3.2. Virtual and Physical Social Networks. Crazy Vote was a social website in Taiwan that provided users with personal web space, such as weblogs and a message board. Due to its unique interface and features for voting on users' portraits, it became the biggest social website for Taiwanese teenagers in 2008 , with more than 20,000 users of ages 15 to 19 . To guide future application development, the company supported a two-month research project to fully understand their users' online activities and expectations of social media [82]. In the study, seven highly active users, who have their own fan clubs and hold social events, and two regular users, participated in both in-depth interviews and contextual inquiries, and the online logs of another 40 highly active users and 40 regular users were sampled at random to understand behavioral patterns in the platform. All qualitative data were analyzed by following grounded theory with Nvivo $[83,84]$. At the same time, contextual design work models and the multilevel social activity model (MLSAM) were used as design research guidelines to capture information and present results [82].

By extending the research scope with both contextual inquires and in-depth interviews, the study shows that teenagers' common processes of making friends are complex, but flexible. In addition, the boundary between online social interaction and actual relationships is very blurred to Taiwanese teenaged online users. Their reason for making new friends on the Internet is simply to expand their interpersonal relationships in the real world. For instance, the interviewees mentioned that they preferred to make friends who live nearby to increase chances of meeting up in person, as society does not encourage teenagers to travel alone. Social issues that are associated with meeting online friends also make teenagers form unique networks to ensure that all members are using their real identities and to later develop real-life confidence in each other.

As shown in Figure 2, the multilevel social activity model also helped us identify three different user types among the website users. First, activity promoters, who were extremely confident and familiar with most social norms and manners on social media, voluntarily held gathering events, established clubs and recruited other users to join their own clubs. Second, followers were willing to participate in social events but had less interest to be a group leader or to organize activities. Third and finally, self-oriented users made up 90\% of the user base, and their activities on the platform were more self-oriented, such as maintaining and updating blogs and photo albums. They seldom visited others' blogs or left messages to others. According to the in-depth interviews, we found that most self-oriented users were either introvert or lacked experience of interaction with unfamiliar people on the Internet. Therefore, we further separated these three 


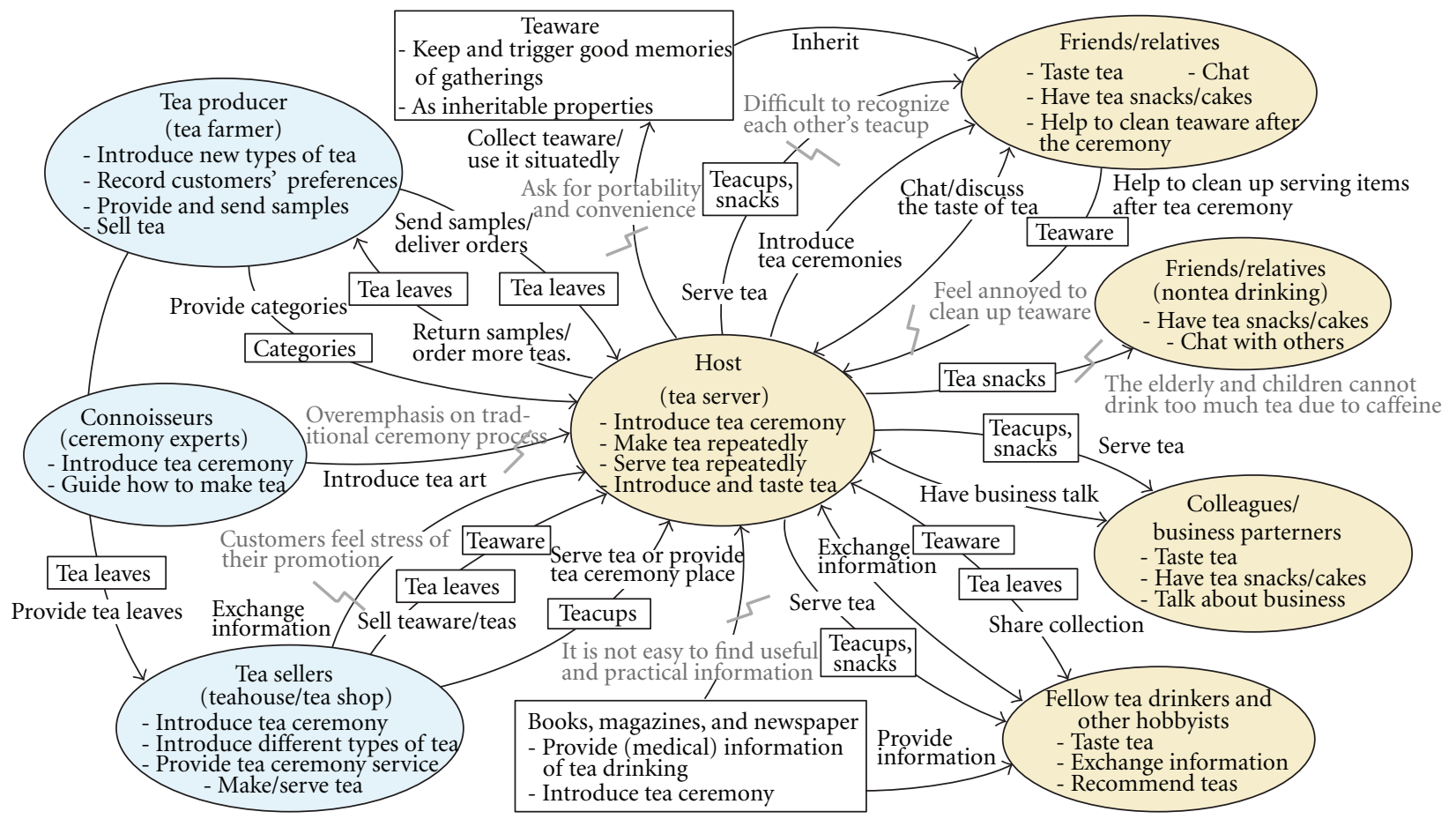

People who actually participate in tea ceremonies

People who are involved in tea ceremonies

(a) The flow model of tea ceremonies

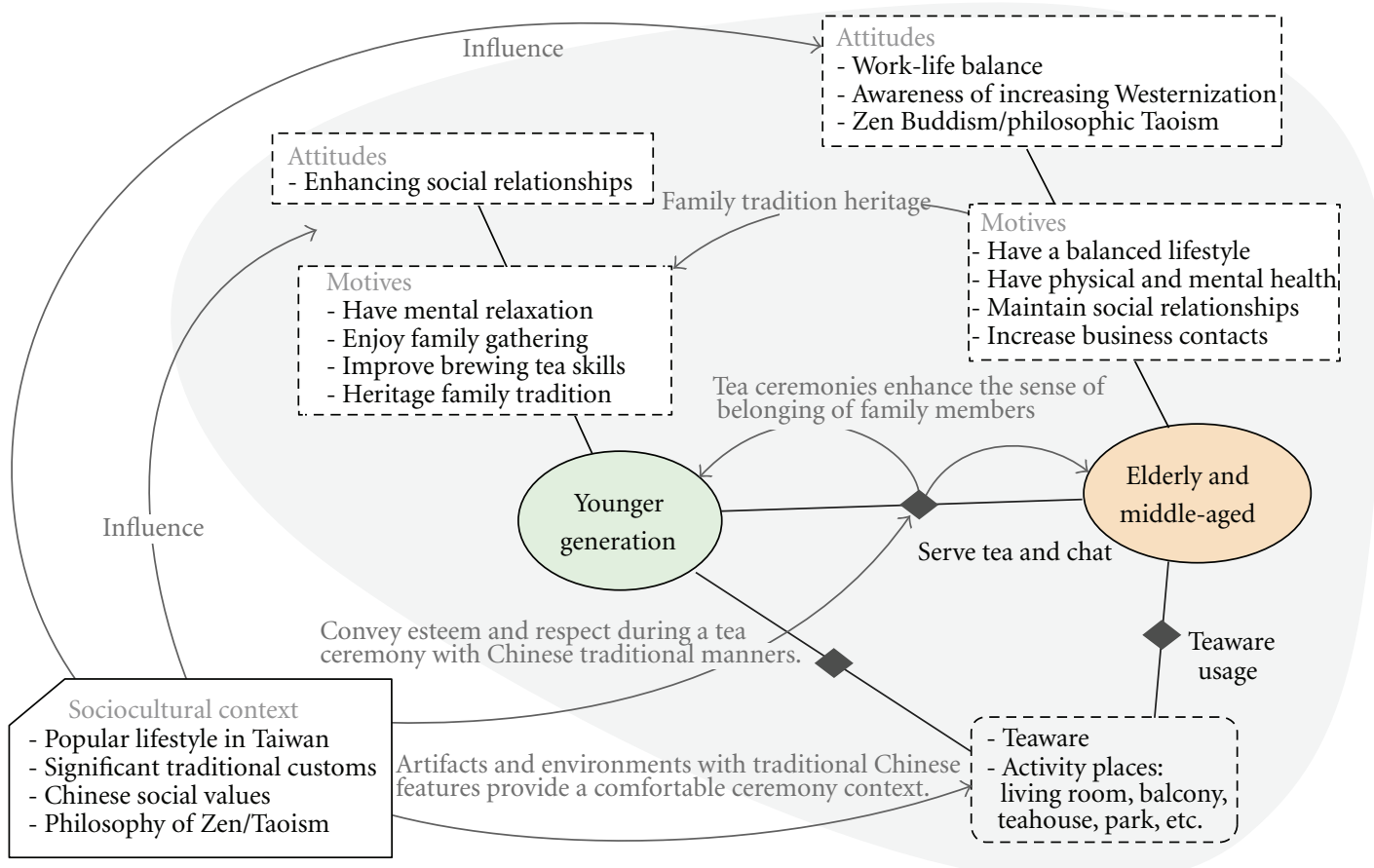

People who are direct consumers of teas and teaware

People who participate in tea ceremonies, but not consumers

(b) The multilevel social activity model of tea ceremonies

Figure 1: Different user types in tea ceremonies. 


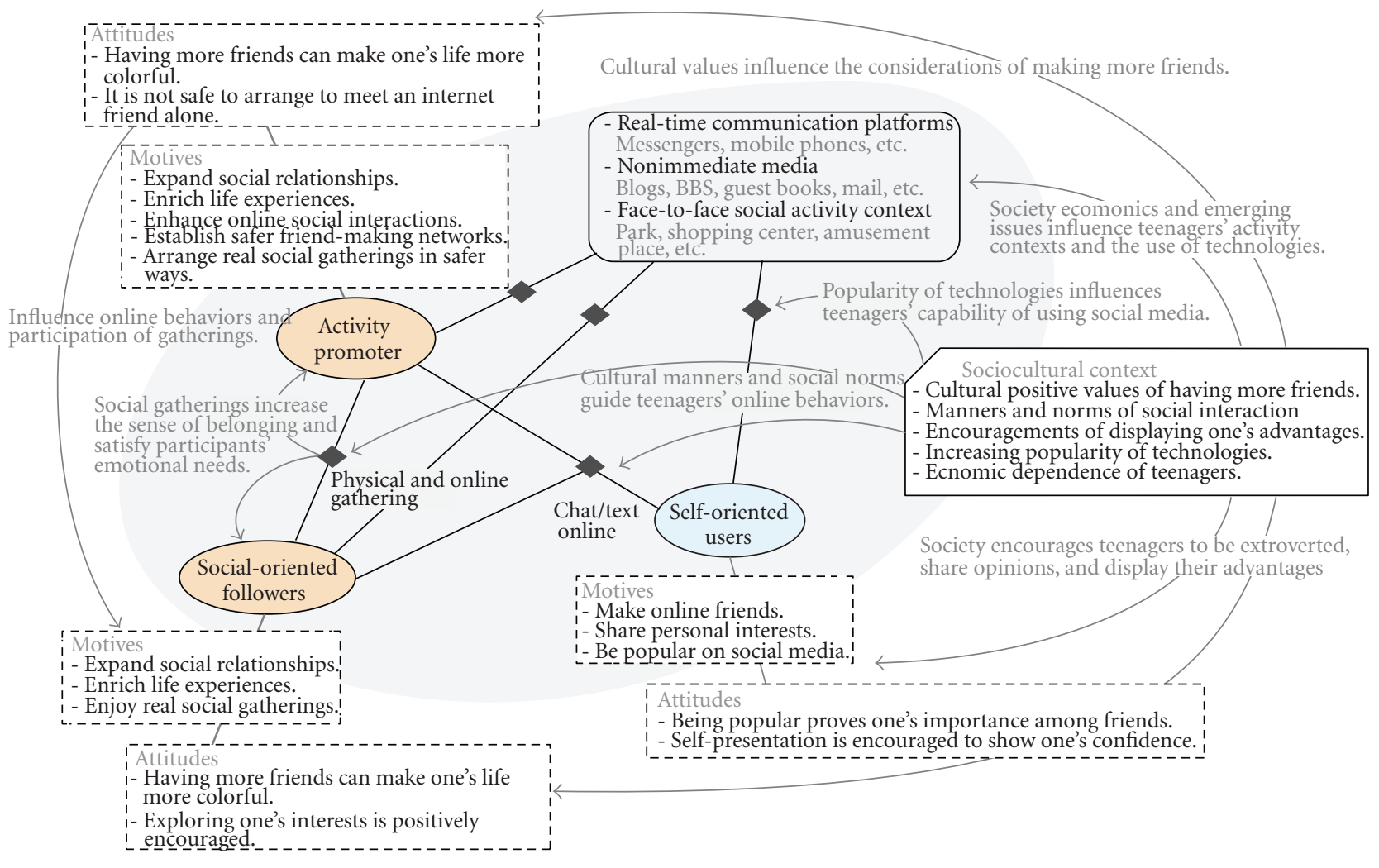

Teenagers who are active users and willingly participate in both virtual and face-to-face social activities

Teenagers whose online activity are more self-oriented, such as maintaining personal blogs and photo albums

FIgURE 2: Diverse user types of social media.

type users, activity promoters, social-oriented followers, and self-oriented users, into to two groups, in which the first group users are more social-oriented (marked in orange in Figure 2) and the second group users are more self-oriented (marked in blue in Figure 2).

Some teen users had a common and well-defined procedure for making friends successfully and efficiently in the Crazy Vote platform, and most users in the socialoriented group were aware of and applied this process. First, nonverbal introductions would take place through the voting system or by sending emoticons to others. Communication would then be initiated by leaving a private message or by visiting and leaving public comments on each other's blogs. People who share similar interests and habits may then exchange other online contact information, such as MSN Messenger or Yahoo! Messenger accounts, and start communicating electronically outside of the Crazy Vote platform. In the end, these online friends may end up talking on mobile phones and meeting up face-to-face.

Rapid expansion of information and communication technology has made young people comfortable with using a wide range of communication platforms. Although taking place in an online environment, the observed process among Taiwanese teenagers for making friends is natural, mature and matches traditional Taiwanese social norms. For instance, the initial use of nonverbal emoticons and "likes" to make others aware of their presence was described in the interviews as a type of "reserved" introduction, similar to a head nod or eye gaze. Young users considered it too aggressive and impolite to suddenly show up and introduce themselves in front of strangers. However, according to both interviews and online tracing, users in the self-oriented group were unaware of this process and fell back on expanding their presentation of themselves in the system. Although both socially and self-oriented users initially shared the common goal of making new friends through the Crazy Vote website, the self-oriented users perceived a difficulty to initiate communication and greet strangers in proper ways, which later caused them to focus on their own blogs.

This study illustrates how more in-depth user research can lead to detailed interaction issues as well as an understanding of sociocultural contexts and their influences on users' motives and behaviors. Such knowledge can also be applied and reused in many different design projects. In addition, understanding users' expectations and abilities helps development teams make better decisions and predict user engagement. For instance, the social-oriented group's capabilities and successful strategies of making friends can 
be applied in social media design to help and guide the other types of users. However, current design research generally focuses on a single platform or a particular environment, which narrows down the research scope and overlooks people's great ability to manipulate different resources to achieve their goals.

\subsection{Large-Scale Services and Cross-Platform Experiences.}

The third case study investigates people's sport watching experiences in Portugal. The design process began with interviews and observations of 20 active sports fans and people who had participated regularly in football watching activities, followed by modeling according to MLSAM [4] and contextual design [40] to understand their experiences, motives, and behaviors in sports watching. On the basis of the models, three personas and 30 different design concepts were then generated in a workshop, and 15 subjects were asked to do card sorting to rank the design concepts. In the design phase, several user experience and service design techniques were applied to define the details of the service system, including scenarios and storyboards, customer journey, usecase analysis, and service design blueprint $[3,17,85,86]$. To gain overall feedback and to improve the systems, formal usability evaluation was conducted with five active football fans, by following collaborative usability inspection, rapid iterative testing, and evaluation and single-subject testing $[87,88]$.

The flow model in Figure 3(a) shows that football fans use laptops and other high-tech devices to enhance their football watching experience at home. Examples include accessing high quality streaming, using a projector, receiving statistical information from websites, and discussing referees and penalties with friends on the Internet. People watching the game at home do so either together with family members or with close friends. Figure 3(b) shows that a football watching activity in a sports bar involves several different types of people in addition to the football fans. This includes peddlers who sell team scarves and jerseys to sport bar customers, the staff, and owner of the bar, and the fans' friends, who despite not being present in the bar interact with bar customers through digital devices.

The multilevel social activity model (Figure 4) distinguishes several types of people by their different motives for participating in sport watching. Primary supporters enjoy watching games, with strong interests in details of the game and high-quality game play. Potential supporters consider football watching primarily as a social activity and their motives for participation are generally derived from primary supporters' interest (e.g., most of our male interviewees mentioned that their girlfriends, wives, and children try to understand the game rules so that they can be more involved in discussions of details together during the game). The other user types, such as bar owners and environment providers, participate in the events because of expectations of business opportunities.

Using the users types identified in the multilevel social activity model, we marked the primary supporters (football fans and community) in orange in Figures 3(a) and 3(b).
Entertainment and media companies have traditionally always targeted their products purely towards primary supporters. Groups marked in blue are not primarily attending the gathering to watch the game, but their greater interest in social interaction nonetheless makes them potential customers of a service. Although they are not the active sports fans, it is possible to include these groups as stakeholders in current or future service design processes to develop better services of greater scope. For instance, bar owners can be clients of media companies, if a service design aims to provide a social entertainment space with complete sport channels and other facilities.

Due to resource and time limitations in this academic project, only primary supporters were selected for the continued development. On the basis of the research findings, two personas were developed who, while both being primary supporters, have very different expectations of the IT product used. As shown in Figure 5, two user portraits were introduced to the development team in the session of brainstorming and decision-making. All project participants and sponsors agreed that the two user portraits were very representative of typical football fans in Portugal. The further customer activity journey, service architecture, scenario, and storyboard were also developed according to our personas' motives, usage of technology, and lifestyles.

Having a clear image of two user types also helped designers and engineers generate correct use cases [89] and prioritize important features in the later stages of prototyping and system development. For instance, the development team discussed different use cases and designed detailed interaction based on service design blueprints, which require correct selections of platforms (channels) for a certain type of users. During implementation, we noticed that our user portraits helped the development team focus on supporting the primary usage situations and helped reduce the complexity and conflicts while supporting cross-platform interactions. Moreover, the two user portraits were also used to plan the usability evaluation and to discuss our service values in user test sessions $[87,88]$.

\section{Static and Dynamic User Portraits}

In this section, we reflect on the three case studies and argue that two types of user information, the dynamic and static attributes, should be captured and included in design research. The suitable methods and techniques to capture this information will also be discussed.

4.1. Dynamic User Information. The cases indicate that there are two types of information that have not been highlighted in general user modeling, profiling, or persona description, yet are important for identifying potential users and predicting their behaviors. The first type of user information is dynamic attributes, such as knowledge space $[14,16]$, age, lifestyles, and IT consumption. The second type is permanent information, such as attitudes, preferences, customs, and appropriate behaviors. 


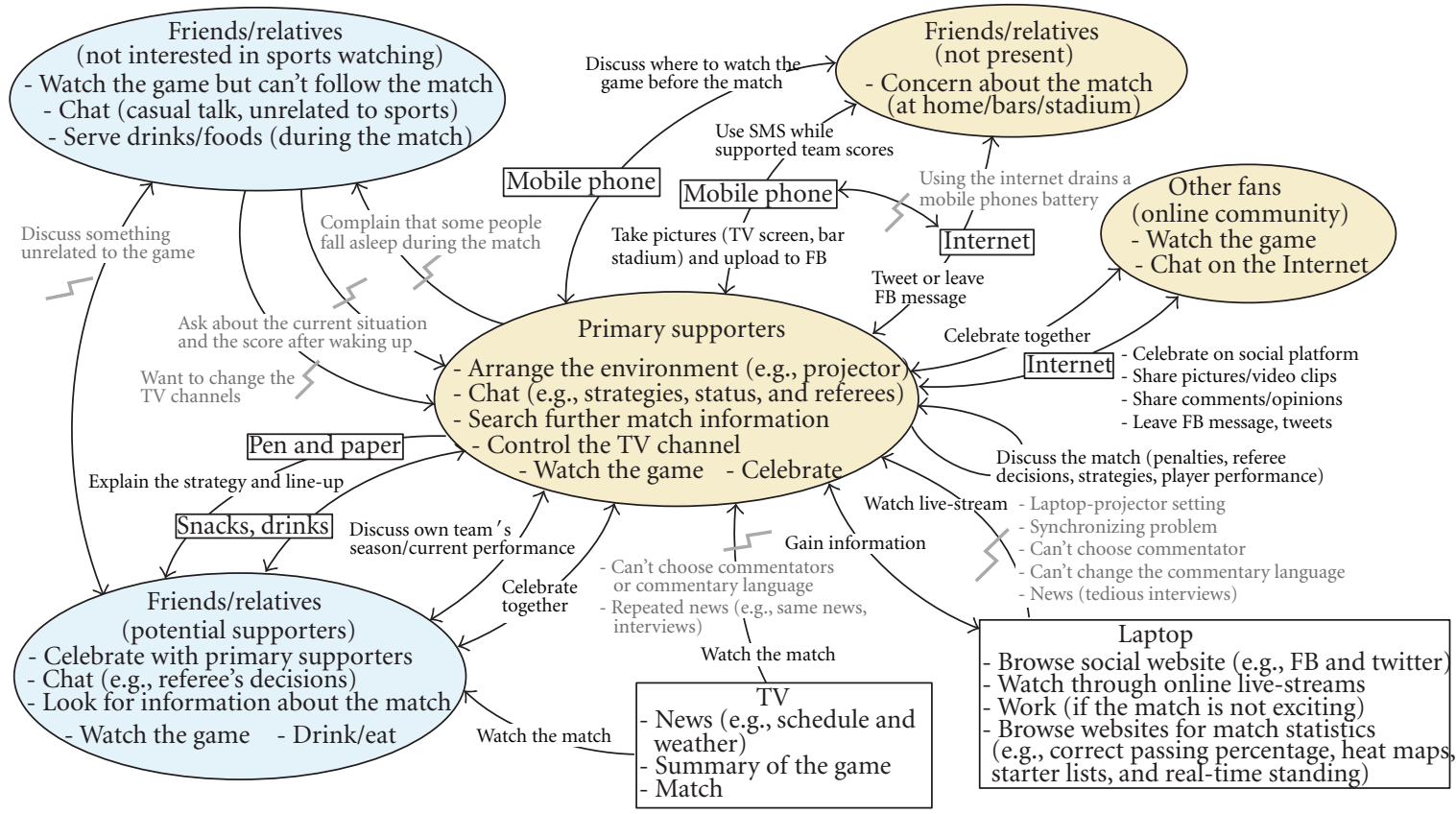

People who are considered as primary users/consumers of most sports related services

People who participate in sport watching, but not fully concentrate on the game

(a) The flow model of football watching at home

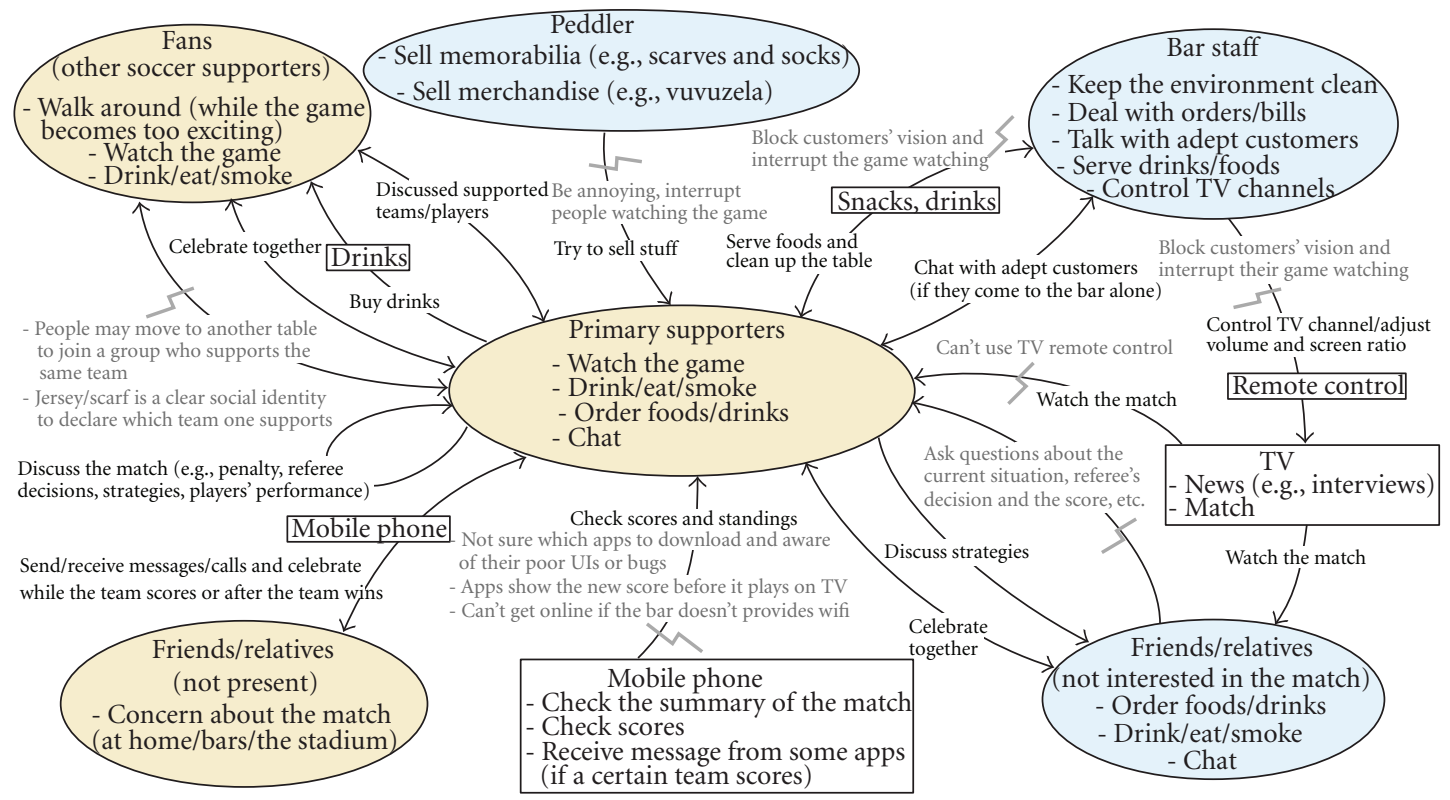

People who are considered as primary users/consumers of most sports related services

People who participate in sport watching, but not fully concentrate on the game

(b) The flow model of football watching at bars

Figure 3: The flow models of sport watching.

The importance of the first type of information emerged from both the case studies of tea ceremonies and of teenagers' social activities. As shown in Figure 1, tea ceremonies are a typical type of traditional social activity that enhances the sense of belonging of family members, and which is passed on from one generation to another. However, from marketing and usage-centered viewpoints, the youth involved in the ceremony are neither consumers nor end users. Due to differences in media usage, they generally receive no information from tea producers or direct-marketing farmers 


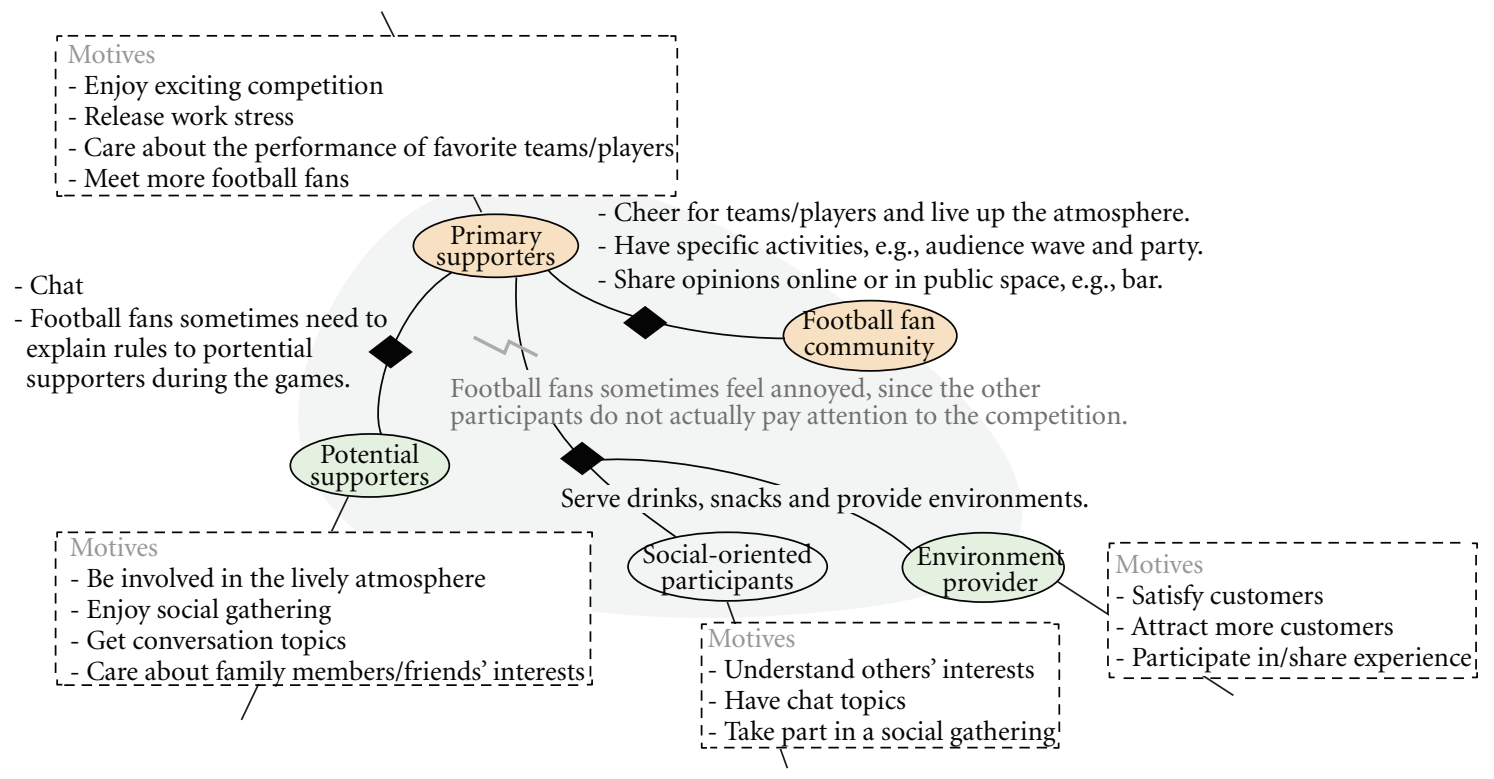

People who are considered as primary users/consumers of most sports related services

- People who are considered as the potential users of our football watching service

FIgURE 4: The multilevel social activity model of sport watching.
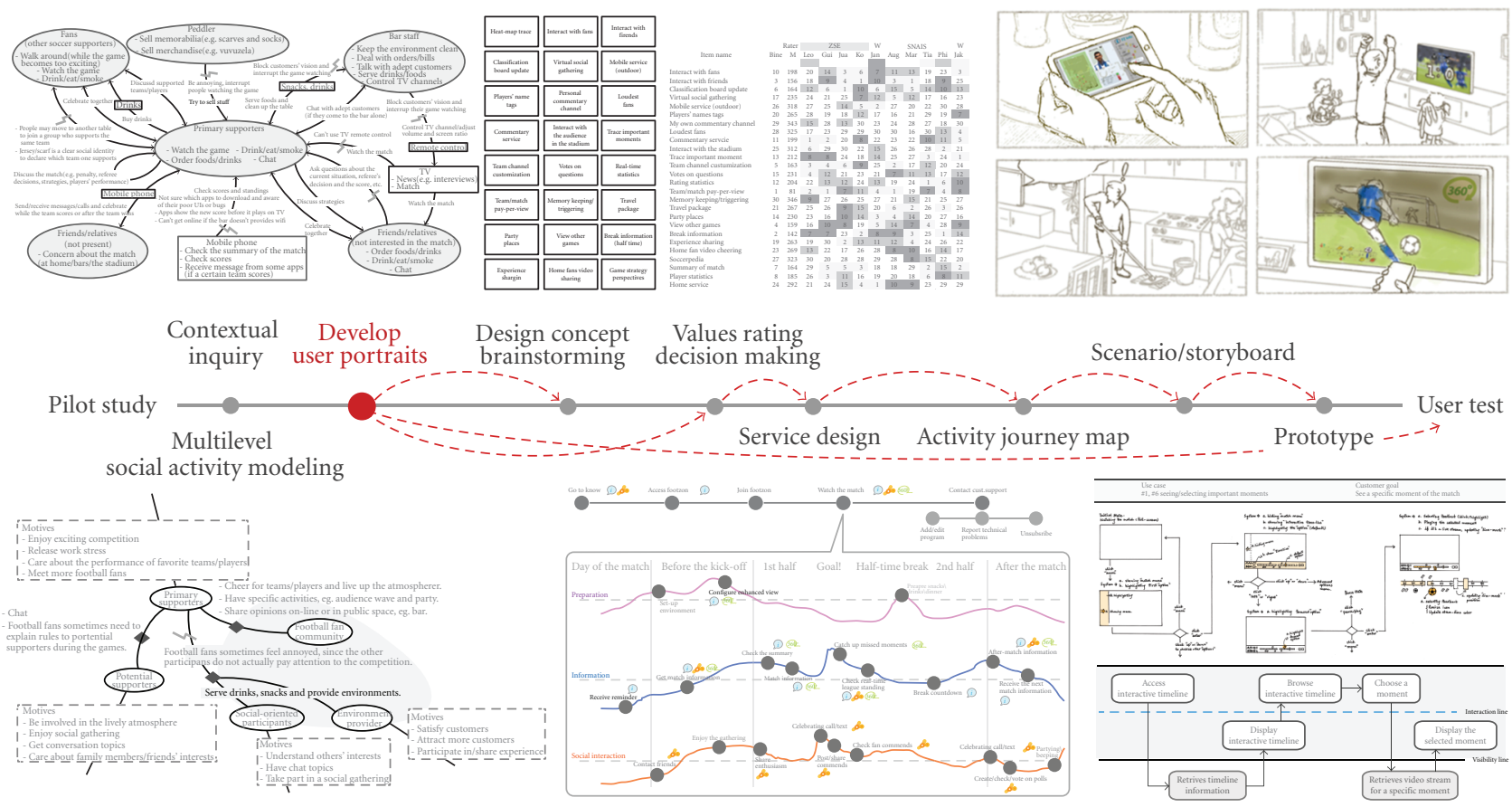

FIGURE 5: The uses of personas in the service design process (red dots).

(which is mainly accessible from magazines, newspaper advertisements, and yellow pages). However, the custom is still inherited, which means that within the predictable future, this young generation will grow to become the target audience of the tea ceremony. In other words, if service designers can identify this potential user group and provide information on the right platform, it is possible to support this cultural inheritance and bridge the gap of media usage among different generations.

In the case study of teenagers' social activities, we found that although teenagers are willing to be active users of technology in their pursuit of new friends, their available modes of communication are often limited by their economic status. As teenagers generally have limited financial resources, most 
IT products and media producers are unwilling to develop services for them. However, the older teenagers will see great improvements in their personal economy in only one or two years, and their great interest and reliance on the Internet will make them the target audience of smart phones, tablets, and other IT products in the near future. This type of information, predicting future audiences, is basic in marketing, but it is often overlooked in design research.

Different from most work in adaptive system development [15], we consider age and IT consumption to be dynamic attributes for user portraits. Traditional systems normally serve a specific type of users. For instance, most educational applications target a user group in a narrowed age range. However, for lifelong learning networks, largescale services, and games with social purposes, the goal is to satisfy a large, diverse audience with long-run usage. Much work has highlighted several dynamic variables in development of technology enhanced learning systems and adaptive games, such as learners' knowledge space, skills, and capabilities $[16,90,91]$. However, we argue that user portraits, profiles, and models should also reflect how people's hypermedia experience, interests, and social behavior change with demographic transitions. Therefore, design research needs to consider predictable shifts in age, lifestyle, economic status, and IT consumption. To capture the dynamic information, surveys, self-documents, and focus groups can provide effective results.

4.2. Static User Information. More permanent and static information, including individuals' preferences, attitudes, and values, has been highlighted in some work of developing personas and user models [11, 14, 17, 28]. However, higherlevel information such as norms, interpersonal relationships, and sociocultural backgrounds, are generally ignored in IT development.

According to multilevel social activity models and flow models of all the case studies, participants in a certain activity can have very similar behaviors, but their concerns, attitudes, and motives may still differ greatly. For instance, in the case study of football watching, we identified that participants' requirements and underlying motives varied, including seeking high-quality watching experiences (primary supporters), seeking detailed information (primary supporters), wanting to discuss the game with others, and enjoying each others' company (potential supporters). However, customer journey maps [92], which are used to describe an activity route of a user and to plan different touch points that characterize user's interactions within the service, cannot reveal such diversity of users' underlying motives and purposes of the activity. Moreover, due to lack of notation of different user types (e.g., differences in technology use), these models cannot fully support development teams when deciding suitable platforms (e.g., touch points and channels in service design). On the basis of our data gathered through multiple methods, we consider that the customer journey map has to be extended into an activity journey map (Figure 6), to reflect users' differing expectations. The map contains three types of customer journeys, representing different users' types and their requirements. The awareness of the differences allows designers to develop reflective and reasonable scenarios and also helps development team prioritize use cases, decide appropriate platforms (channels), and plan user testing.

In addition, understanding of high-level information such as values, attitudes, and sociocultural background is easily taken for granted and therefore ignored in design research. Mulwa et al. [91] have listed twenty-one different user features for developing adaptive educational systems in the literature from 1996 to 2008, but none of these variables reflects values, social behaviors, and activity contexts. Most user experience design and HCI research methods can reveal detailed usage situations and users' cognitive characteristics, but have difficulty identifying meanings and norms behind activities. For instance, teenagers' friend-making processes on the Internet still follow the cultural manners that apply in their daily life. Additionally, in the case study of tea ceremonies some participants considered the ceremony to be part of East Asian culture and feel responsible to maintain this tradition.

4.3. Identify Invisible Users and Portray Users. On the basis of the studies of different social activities in different countries, we have discussed the strengths and weaknesses of different research methods. To better support rapid design research in most IT development, common user-centered design methods and frameworks provide a cheaper and more efficient way to account for the behaviors and interests of target users. However, in design for supporting communication, social purpose, and larger-scale services, we recommend to apply multiple research methods to gain deeper insights into the contexts and to identify potential and invisible users. Therefore, we propose the following process.

(1) Focus on a certain activity: as a pilot study, researchers can choose a representative activity and apply common context-, user-, or usage-centered research methods to capture events in great detail. In this stage, people who actually participated in the activity, objects, media, environmental circumstances, and interactions are identified.

(2) Extend the context: the goal of this stage is to identify the flow of information and find the social network that is related to the activity in the pilot study. Through an iterative process, researchers can identify how both central and peripheral events and people are connected with each other and associated with the main activity. To avoid missing important details, we suggest applying long-term ethnographic approaches, such as interviews, shadowing, behavior tracing, and self-documents.

(3) Recognize participants: in this stage, all people's goals, roles, interests, and participation in the target activity should be clarified. This information can help researchers to further distinguish different user types and identify potential users.

(4) Selection and focus: a workshop or a focus group in this stage can help development teams decide which 


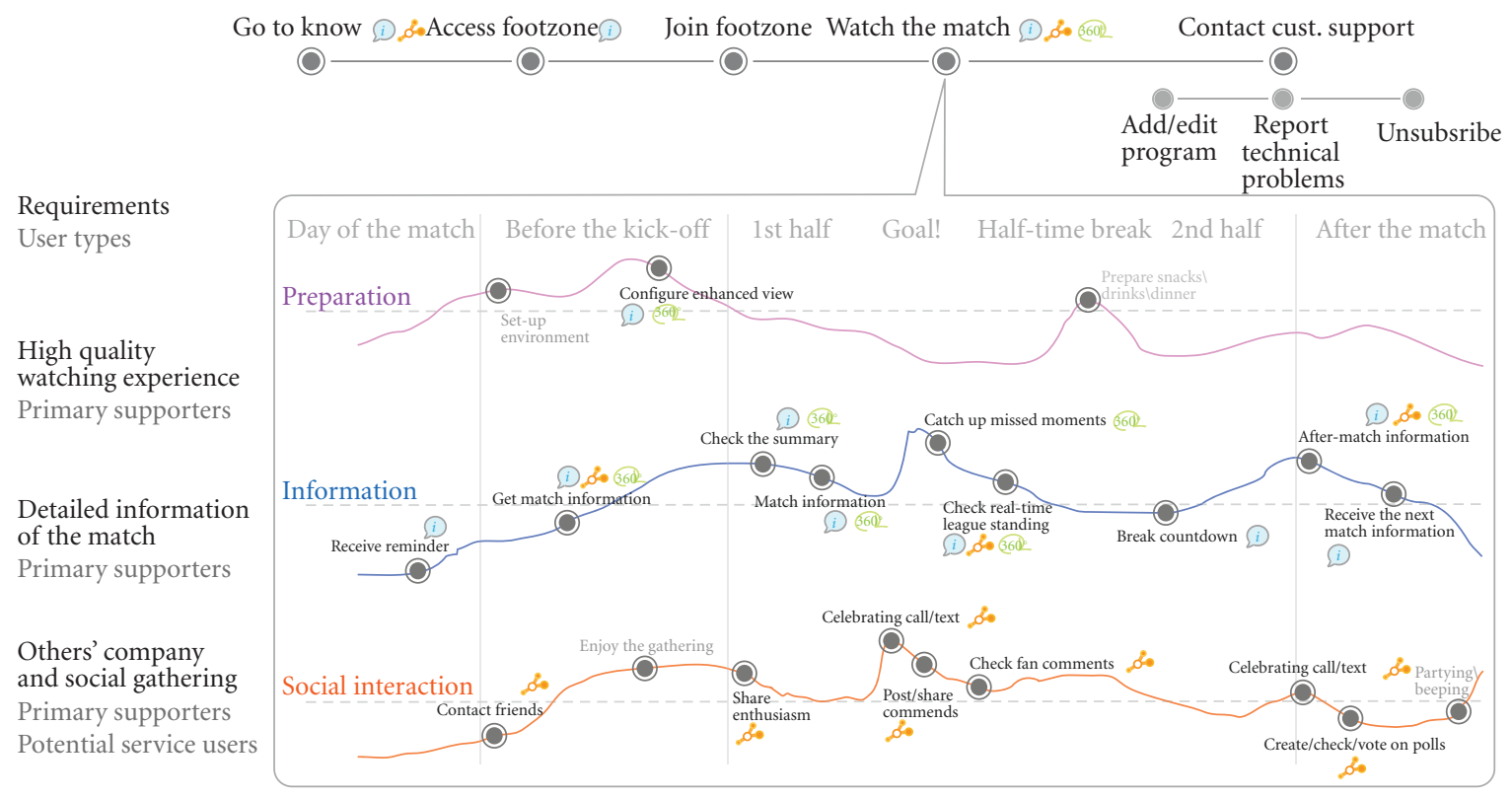

FIGURE 6: The activity journey map.

types of users should become the target audience and to set clear priorities for design development. Once the user types are selected, surveys and indepth interviews can be used to capture in-depth information about these selected users.

To make the research results serve the same purpose as user models and personas, both dynamic and static information need to be highlighted while portraying the users. First, the portrait (it can be presented as a model or a document) should contain basic demographic information, such as gender, interests, and preferences. However, different from normal user profiling, we highly recommend that researchers also include dynamic user attributes that help predict how users' behaviors will change with shifts in their demographic data. For example, predictable increases in income are associated with transitions from a teenage life to maturity and result in changes in IT consumption and use. The second type, static information, is about higher-level concerns, including users' attitudes, motives, beliefs, and their sociocultural background. As mentioned above, this information is permanent and can enrich design solutions and can help evaluate designs in different phases of design cycles and in different projects.

\section{Discussion}

Through contextual design flow modeling, multilevel social activity modeling, and traditional ethnographic techniques, we have shown that there are different types of invisible users, who are involved in social activities, but are neither direct product users nor customers. In the case of a tea ceremony, the young generation, who has inherited tea-drinking habits from their parents, is not considered as a target audience of the traditional tea industry and can only access very limited information. In the case of football watching, potential supporters represent a large group of people who participate in the activities regularly because their children, parents, friends, or colleagues are football fans, and they are willing to share these interests. However, most entertainment and media companies have only considered providing services for football fans. On the basis of what we learn from the three studies, it is argued that discovering all user types and presenting static and dynamic user portraits will bring many long-term benefits for systems and services.

(i) Identify potential users: in the three cases, we have shown that extending the research scope can help gain the necessary understanding of complex social interactions and social contexts and discover potential and indirect end users. We consider this to be significant understanding for serious games and technology enhanced education systems.

(ii) Support diverse and large user groups: development teams can predict new needs and expectations of future target audiences by identifying predictable changes in users' IT-product usage and economic status. Furthermore, identifying user types and motives can help designers propose more reasonable design solutions, satisfy users' different needs, and develop more adaptive systems.

(iii) Support multiple projects: compared to the outputs of usability and activity research, sociocultural information such as attitudes, values, and norms is more permanent in time and is valid across different media and physical spaces. Therefore, this type of knowledge can continuously be reused and reapplied in different projects. For instance, both the first and second studies have shown people's daily life and online activities are driven by norms and 
sociocultural values, which can always be considered in social media development.

As discussed in Section 2, most design research frameworks developed in HCI and related IT development generally do not focus on identifying user types or portraying users with the important dynamic and permanent attributes highlighted in the previous section. The design case studies have shown that ethnographic approaches, in-depth interviews, flow modeling, and multilevel social activity modeling can help development teams gain deeper insights, but that these methods are also time consuming and may require highly experienced researchers. Complex social media, service, and serious games development is challenging due to involving diverse user types, uncertain activity goals, and complex social interactions. Therefore, we argue that more comprehensive design research with a broader scope is needed to gain deeper insights, which can continue benefiting different phases in an iterative design cycle and help develop more adaptive and thoughtful systems.

\section{Conclusion}

Traditional user modeling mainly focuses on humancomputer interaction. Although the traditional models reveal detailed information of the ways that people interact with systems, they are incapable of capturing many factors and contexts critical to design for social interaction, adaptive systems, and serious games. Personas, on the other hand, represent rich information about users' lifestyles and attitudes and bring various benefits to design practices. However, there is little consensus regarding suitable methodology for how to develop reliable and representative personas. Both user modeling and personas narrow down their audience groups by overlooking activity contexts. User modeling only focuses on direct users of systems, and personas mainly represent target consumers, rather than describing the many participants involved in the activities.

In this research, we compare the strengths and weaknesses of different design and research methods. We, first, present additional types of user information that were gained through application of multiple research methods. Second, we discuss the benefits of having this information in a design process, including being able to identify invisible users, increase awareness of different user types, and develop more informative and representative user portraits. We also suggest suitable research methods in each project phase and list the important information needed to develop user portraits. In addition, we provide many examples through our cases to illustrate how the knowledge of user types can be applied in a design process, in particular in the development of large-scale services, adaptive systems, and serious games. We are aware that the cost of applying long-term ethnographic approaches and multiple modeling strategies to gain these insights is high. However, following scientific approaches can contribute high-quality and reliable user portraits with significant dynamic and static user information, which can later be reused and reapplied in different future projects.

\section{References}

[1] K. Lynch, The Image of the City, Technology Press and Harvard University Press, Cambridge, Mass, USA, 1960.

[2] J. Zeisel, Inquiry by Design: Tools for Environment-Behavior Research, Cambridge University Press, 1984.

[3] B. Laure, Ed., Design Research: Methods and Perspectives, MIT Press, Cambridge, Mass, USA, 2003.

[4] K. H. Huang and Y. S. Deng, "Social interaction design in cultural context: a case study of a traditional social activity," International Journal of Design, vol. 2, no. 2, pp. 81-96, 2008.

[5] K. Holtzblatt and S. Jones, "Contextual inquiry: a participatory technique for system design," in Participatory Design: Principles and Practices, D. Schuler and A. Namioka, Eds., pp. 177-210, Lawrence Erlbaum Associates, Hillsdale, NJ, USA, 1993.

[6] F. Kensing and J. Blomberg, "Participatory design: issues and concerns," Computer Supported Cooperative Work, vol. 7, no. 3-4, pp. 167-185, 1998.

[7] J. Preece, Y. Rogers, and H. Sharp, Interaction Design: Beyond Human-Computer Interaction, John Wiley \& Sons, New York, NY, USA, 2000.

[8] E. Arias, H. Eden, G. Fischer, A. Gorman, and E. Scharff, "Transcending the individual human mind: creating shared understanding through collaborative design," ACM Transactions on Computer-Human Interaction, vol. 7, no. 1, pp. 84$113,2000$.

[9] A. Kobsa, J. Koenemann, and W. Pohl, "Personalised hypermedia presentation techniques for improving online customer relationships," The Knowledge Engineering Review, vol. 16, no. 2, pp. 111-155, 2001.

[10] K. H. Huang, N. Nunes, L. Nobrega, L. Constantine, and M. Chen, "Hammering model: designing usable modeling tools," in Human-Computer Interaction-INTERACT, P. Campos et al., Ed., vol. 2011, pp. 537-554, Springer, 2011.

[11] P. Brusilovsky, "Adaptive hypermedia," User Modelling and User-Adapted Interaction, vol. 11, no. 1-2, pp. 87-110, 2001.

[12] G. Fischer, "User modeling in human-computer interaction," User Modelling and User-Adapted Interaction, vol. 11, no. 1-2, pp. 65-86, 2001.

[13] M. Montaner, B. López, and J. L. De La Rosa, "A taxonomy of recommender agents on the internet," Artificial Intelligence Review, vol. 19, no. 4, pp. 285-330, 2003.

[14] P. Karampiperis and D. Sampson, "Adaptive learning resources sequencing in educational hypermedia systems," Educational Technology and Society, vol. 8, no. 4, pp. 128-147, 2005.

[15] J. Hothi and W. Hall, "An evaluation of adapted hypermedia techniques using static user modeling," in Proceedings of the 2nd Workshop on Adaptive Hypertext and Hypermedia, pp. 4550, Pittsburgh, Pa, USA, 1998.

[16] N. Henze and W. Nejdl, "A logical characterization of adaptive educational hypermedia," New Review of Hypermedia and Multimedia, vol. 10, no. 1, pp. 77-113, 2004.

[17] E. M. Raybourn, "Applying simulation experience design methods to creating serious game-based adaptive training systems," Interacting with Computers, vol. 19, no. 2, pp. 206214, 2007.

[18] A. Cooper, The Inmates are Running the Asylum, SAMS, Transcending, Ind, USA, 2004.

[19] F. Long, "Real or imaginary: the effectiveness of using personas in product design," in Proceedings of the Irish Ergonomics Society Annual Conference, pp. 1-10, Dublin, Ireland, 2009.

[20] B. Pike, "Persona management," Computer Fraud and Security, vol. 2010, no. 11, pp. 11-15, 2010. 
[21] T. Miaskiewicz and K. A. Kozar, "Personas and user-centered design: how can personas benefit product design processes?" Design Studies, vol. 32, no. 5, pp. 417-430, 2011.

[22] A. N. Antle, "Child-personas: fact or fiction?" in Proceedings of the Conference on Designing Interactive Systems (DIS '06), pp. 22-30, New York, NY, USA, June 2006.

[23] A. N. Antle, "Child-based personas: need, ability and experience," Cognition, Technology and Work, vol. 10, no. 2, pp. 155$166,2008$.

[24] R. Chen and X. Wang, "Conceptualizing tangible augmented reality systems for design learning," in Proceedings of the Design Computing and Cognition '08, J. S. Gero and A. K. Goel, Eds., pp. 697-712, Springer, 2008.

[25] L. Suchman, Plans and Situated Actions: The Problem of Human-Machine Communication, Cambridge University Press, Cambridge, UK, 1987.

[26] M. S. Ackerman, "Intellectual challenge of CSCW: the gap between social requirements and technical feasibility," $\mathrm{Hu}$ man-Computer Interaction, vol. 15, no. 2-3, pp. 179-203, 2000.

[27] G. Fitzpatrick, The Locales Framework, Kluwer Academic Publishers, Amsterdam, The Netherlands, 2003.

[28] H. Drachsler, H. G. K. Hummel, and R. Koper, "Personal recommender systems for learners in lifelong learning networks: the requirements, techniques and model," International Journal of Learning Technology, vol. 3, no. 4, pp. 404-423, 2008.

[29] R. Koper and P. Sloep, Learning Networks: Connecting People, Organizations, Autonomous Agents and Learning Resources to Establish the Emergence of Effective Lifelong Learning, Heerlen, Open University of Netherlands, Amsterdam, The Netherlands.

[30] K. Rönkkö, “An empirical study demonstrating how different design constraints, project organization and contexts limited the utility of personas," in Proceedings of the 38th Annual Hawaii International Conference on System Sciences, p. 220, Washington, DC, USA, January 2005.

[31] C. N. Chapman and R. P. Milham, “The personas' new clothes: methodological and practical arguments against a popular method," in Proceedings of the 50th Annual Meeting of the Human Factors and Ergonomics Society (HFES '06), pp. 634636, October 2006.

[32] C. N. Chapman, E. Love, R. P. Milham, P. Elrif, and J. L. Alford, "Quantitative evaluation of personas as information," in Proceedings of the 52nd Human Factors and Ergonomics Society Annual Meeting (HFES '08), pp. 1107-1111, September 2008.

[33] S. Portigal, "Persona non grata," Interactions, vol. 15, no. 1, pp. 72-73, 2008.

[34] K. H. Huang, C. H. Wuang, and H. L. Chong, "FootPal: build social rivalries around maintainable walking habits," in Proceedings of the Extended Abstracts on Human Factors in Computing Systems (CHI '10), ACM, New York, NY, USA, 2010.

[35] C. H. Wu, T. F. Wu, Y. H. Chou et al., "HappyFeet! Influencing at the turning points: walking or scooter ride for shortdistance journey?" in Proceedings of the 12th International Conference on Human Computer Interaction with Mobile Devices and Services, pp. 463-466, ACM, New York, NY, USA, 2010.

[36] F. W. Tung and Y. S. Deng, "Designing social presence in elearning environments: testing the effect of interactivity on children," Interactive Learning Environments, vol. 14, no. 3, pp. 251-264, 2006.

[37] F. W. Tung and Y. S. Deng, "Increasing social presence of social actors in e-learning environments: effects of dynamic and static emoticons on children," Displays, vol. 28, no. 4-5, pp. 174-180, 2007.
[38] B. Glaser and A. Strauss, The Discovery of Grounded Theory: Strategies of Qualitative Research, Weidenfeld and Nicolson, 1967.

[39] A. Strauss and J. Corbin, Basic of Qualitative Research: Grounded Theory Procedures and Techniques, Sage, 1990.

[40] H. Beyer and K. Holtzblatt, Contextual Design: Defining Customer-Centered Systems, Morgan Kaufmann Publishers, 1998.

[41] H. Garfinkel, Studies in Ethnomethodology, Prentice-Hall, Englewood Cliffs, NJ, USA, 1967.

[42] J. Heritag, Garfinkel and Ethnomethodology, Polity Press, Cambridge, Mass, USA, 1984.

[43] M. D. LeCompte and J. P. Goetz, "Problems of reliability and validity in ethnographic research," Review of Educational Research, vol. 52, no. 1, pp. 31-60, 1982.

[44] E. G. Guba and Y. S. Lincoln, Naturalistic Inquiry, Sage, Newbury Park, Calif, USA, 1985.

[45] J. W. Creswell and D. L. Miller, "Determining validity in qualitative inquiry," Theory into Practice, vol. 39, no. 3, pp. 124-130, 2000.

[46] A. Strauss, Qualitative Analysis for Social Scientists, Cambridge, University Press, Cambridge, UK, 1987.

[47] L. Smircich, "Is the concept of culture a paradigm for understanding organizations and ourselves," in Organizational Culture, P. J. Frost, L. F. Moore, M. R. Louis, C. C. Lundberg, and J. Martin, Eds., pp. 55-72, Sage, Thousand Oaks, Calif, USA, 1985.

[48] R. E. Quinn and M. R. McGrath, "The transformation of organizational cultures: a competing values perspective," in Organizational Culture, P. J. Frost, L. F. Moore, M. R. Louis, C. C. Lundberg, and J. Martin, Eds., pp. 315-334, Sage, Thousand Oaks, Calif, USA, 1985.

[49] D. M. Rousseau, "Assessing organizational culture: the case for multiple methods," in Organizational Climate and Culture, B. Schneider, Ed., pp. 153-192, Jossey-Bass, San Francisco, Calif, USA, 1990.

[50] E. H. Schein, Organisational Culture and Leadership, JosseyBass, San Francisco, Calif, USA, 1992.

[51] M. Erez and E. Gati, "A dynamic, multi-level model of culture: from the micro level of the individual to the macro level of a global culture," Applied Psychology, vol. 53, no. 4, pp. 583-598, 2004.

[52] C. Handy, Understanding Organizations, Penguin, Harmondsworth, UK, 1976.

[53] D. Hellriegel and W. Slocum, Organizational Behavior, SouthWestern, 2007.

[54] R. Harrison, "Understanding your organization's character," Harvard Business Review, vol. 50, no. 3, pp. 119-128, 1972.

[55] L. Goodstein, T. N. Nolan, and J. W. Pfeiffer, Applied Strategic Planning, McGraw-Hill, New York, NY, USA, 1993.

[56] A. Brown, Organisational Culture, Pitman, London, UK, 1998.

[57] T. Deal and A. Kennedy, Corporate Cultures: The Rites and Rituals of Corporate Life, Penguin Books, London, UK, 1982.

[58] E. T. Hall, The Hidden Dimension, Doubleday \& Company, New York, NY, USA, 1967.

[59] E. T. Hall, Beyond Culture, Anchor Books, New York, NY, USA, 1977.

[60] G. Hofstede, Culture's Consequences: Comparing Values, Behaviors, Institutions and Organizations across Nations, Sage, Thousand Oaks, Calif, USA, 2001.

[61] G. Hofstede, Cultures and Organizations: Software of the Mind, McGraw-Hill, New York, NY, USA, 1991. 
[62] F. Trompenaars and C. Hampden-Turner, Riding the Waves of Culture Understanding Cultural Diversity in Business, Nicholas Brearley, London, UK, 1997.

[63] E. H. Schein, "Organizational culture," American Psychologist, vol. 45, no. 2, pp. 109-119, 1990.

[64] J. Martin and D. Meyerson, "Organizational cultures and the denial, channeling, and acknowledgement of ambiguity," in Managing Ambiguity and Change, L. R. Pondy, R. J. Boland, and H. Thomas, Eds., Wiley, New York, NY, USA, 1988.

[65] K. R. Thompson and F. Luthans, "Organizational culture: a behavioural perspective," in Organizational Climate and Culture, B. Schneider, Ed., Jessey-Bass, Oxford, UK, 1990.

[66] H. Spencer-Oatey, Culturally Speaking: Managing Rapport Through Talk Across Cultures, Continuum, London, UK, 2000.

[67] M. Krumbholz and N. Maiden, "How culture might impact on the implementation of enterprise resource planning packages," in Advanced Information Systems Engineering, B. Wangler and L. Bergman, Eds., pp. 279-293, Springer, 2000.

[68] D. A. Norman, The Design of Everyday Things, Basic Books, New York, NY, USA, 1988.

[69] G. Ritzer and D. Goodman, Sociological Theory, McGraw-Hill, London, UK, 2004.

[70] C. Wasson, "Ethnography in the field of design," Human Organization, vol. 59, no. 4, pp. 377-388, 2000.

[71] J. Whiteside, J. Bennett, and K. Holzblatt, "Usability engineering: our experience and evolution," in Handbook of Human Computer Interaction, M. Helander, Ed., North Holland, New York, NY, USA, 1998.

[72] D. Wixon, K. Holtzblatt, and S. Knox, "Contextual design: an emergent view of system design," in Proceedings of the Conference of Human Factors in Computing Systems, pp. 329336, ACM, New York, NY, USA, 1990.

[73] Y. Engeström, "Activity theory as a framework for analyzing and redesigning work," Ergonomics, vol. 43, no. 7, pp. 960-974, 2000.

[74] P. D. Rothstein, "a (x 4): a user-centered method for designing experience," in Proceedings of the Design Education. Industrial Designers Society of America (IDSA '01), 2001.

[75] V. Kumar and P. Whitney, "Faster, cheaper, deeper user research," Design Management Journal, vol. 14, no. 2, pp. 5057, 2003.

[76] B. Nardi, "Studying context: a comparison of Activity Theory, situated action models, and distributed cognition," in Context and Consciousness-Activity Theory and Human-Computer Interaction, B. Nardi, Ed., MIT Press, Cambridge, Mass, USA, 1996.

[77] D. H. Jonassen and L. Rohrer-Murphy, "Activity theory as a framework for designing constructivist learning environments," Educational Technology Research and Development, vol. 47, no. 1, pp. 61-79, 1999.

[78] Y. Engeström, "Learning by Expanding. An Activity-theoretical approach to developmental research," 2011, http://communication.ucsd.edu/MCA/Paper/Engestrom/expanding/toc .htm.

[79] M. Fjeld, K. Lauche, M. Bichsel, F. Voorhorst, H. Krueger, and M. Rauterberg, "Physical and virtual tools: activity theory applied to the design of groupware," Computer Supported Cooperative Work, vol. 11, no. 1-2, pp. 153-180, 2002.

[80] S. L. Bryant, A. Forte, and A. Bruckman, "Becoming Wikipedian: transformation of participation in a collaborative online encyclopedia," in Proceedings of the International ACM SIGGROUP Conference on Supporting Group Work (GROUP '05), pp. 1-10, November 2005.
[81] K. H. Huang, An Integrated Research Approach for Social Interaction Design, National Chiao Tung University, Hsinchu, Taiwan, 2012.

[82] K. H. Huang, H. C. You, and Y. S. Deng, "Clubs forming on CrazyVote- the blurred social boundary between online communities and the real world," International Journal of Social and Human Sciences, vol. 3, pp. 693-703, 2009.

[83] G. R. Gibbs, Qualitative Data Analysis: Explorations with NVivo, Buckingham, Philadelphia, Pa, USA, 2002.

[84] P. Bazeley, Qualitative Data Analysis with NVivo, Sage, London, UK, 2007.

[85] G. L. Shostack, "Designing services that deliver," Harvard Business Review, vol. 62, no. 1, pp. 133-139, 1984

[86] M. J. Bitner, A. L. Ostrom, and F. N. Morgan, "Service blueprinting: a practical technique for service innovation," California Management Review, vol. 50, no. 3, pp. 66-94, 2008.

[87] J. Nielsen and R. L. Mack, Eds., Usability Inspection Methods, JohnWiley \& Sons, 1994.

[88] L. Constantine, "Peer reviews for usability," Cutter IT Journal, vol. 18, no. 1, pp. 5-13, 2005.

[89] A. Cockburn, Writing Effective Use Cases, Addison-Wesley, 2001.

[90] D. Charles, M. McNeill, M. McAlister et al., "Player-centred game design: player modelling and adaptive digital games," in Proceedings of the Conference Changing Views-Worlds in Play (DiGRA '05), pp. 285-298, British Columbia, 2005.

[91] C. Mulwa, S. Lawless, M. Sharp, I. Arnedillo-Sanchez, and V. Wade, "Adaptive educational hypermedia systems in technology enhanced learning: a literature review," in Proceedings of the ACM Conference on Information Technology Education (SIGITE'10), pp. 73-84, January 2010.

[92] C. Voss and L. Zomerdijk, "Innovation in experiential services-an empirical view," in Innovation in Services, Department of Trade and Industry, Ed., pp. 97-134, DTI, London, UK, 2007. 

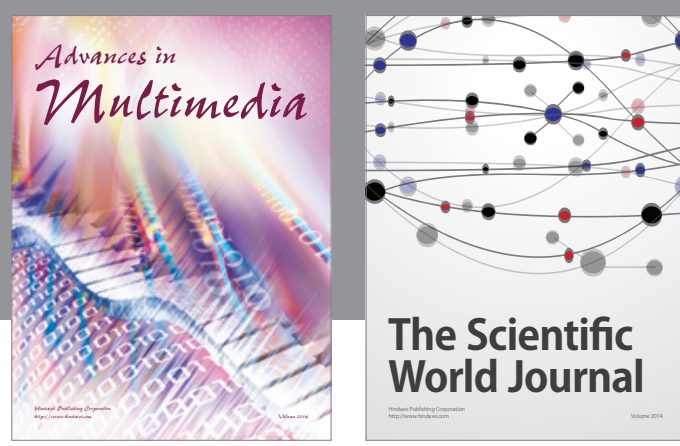

The Scientific World Journal
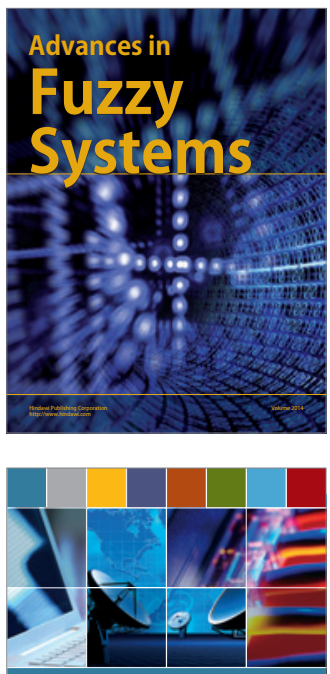

Computer Networks and Communications
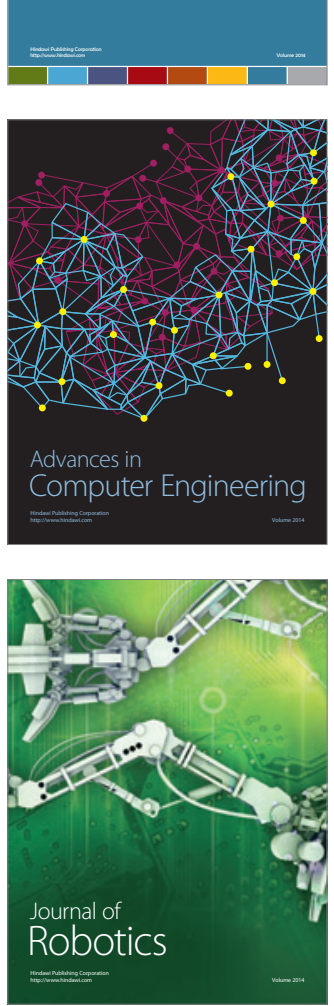
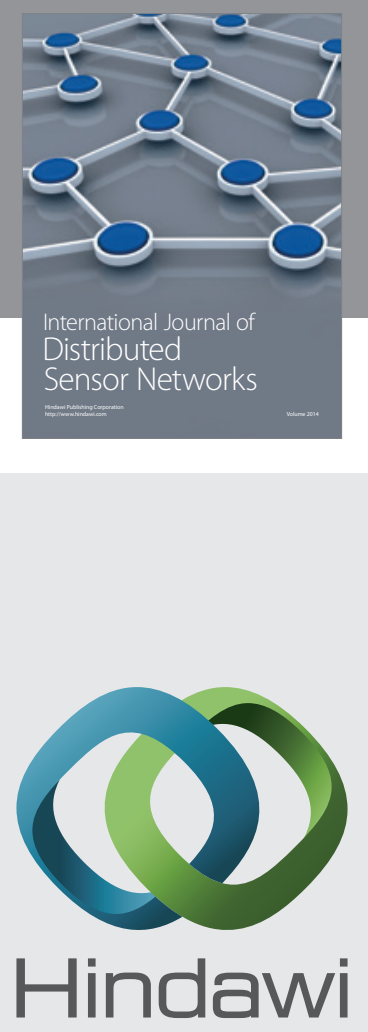

Submit your manuscripts at

http://www.hindawi.com
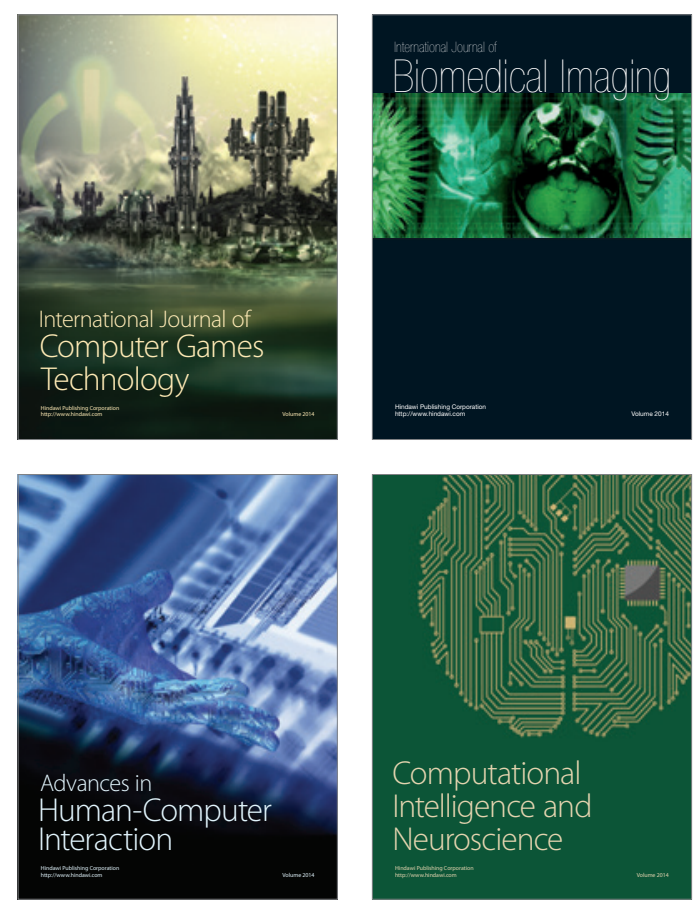
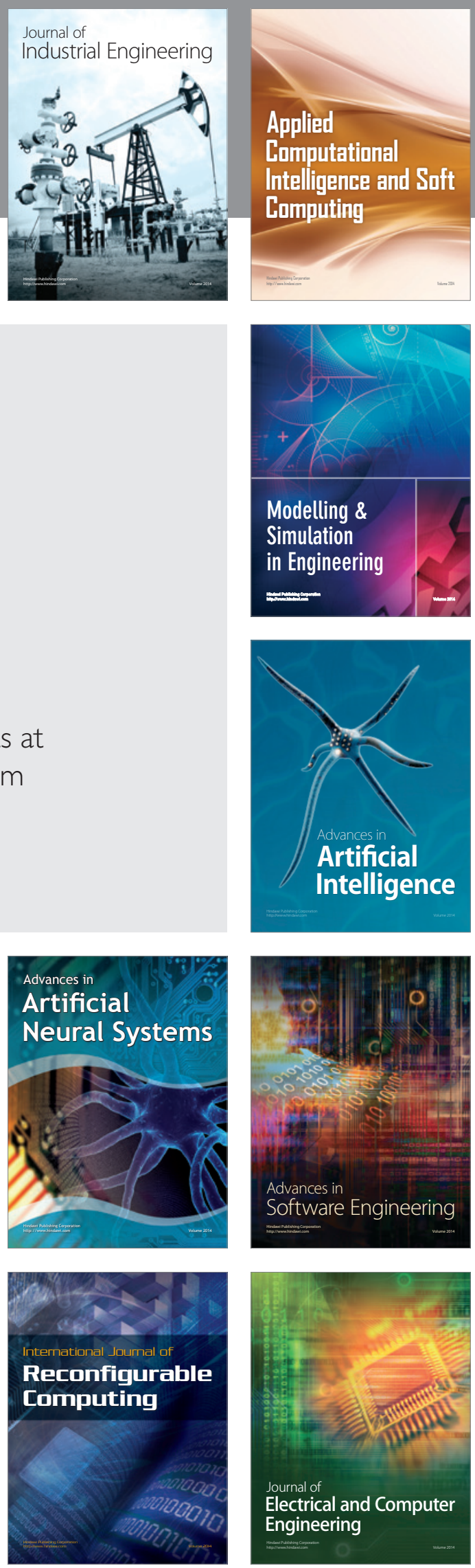